\title{
EFFICIENT USE OF CLOSED-FORM GREEN'S FUNCTIONS FOR THREE-DIMENSIONAL PROBLEMS INVOLVING MULTILAYERED MEDIA
}

\author{
$\begin{array}{cc}\text { M. I. Aksun* } & \text { R. Mittra } \\ \text { Electrical \& Electronics Engineering Electromagnetic Communication Laboratory }\end{array}$ \\ Bilkent university \\ Ankara 06533, TURKEY \\ Dept. of Electrical \& Computer Engineering \\ University of Illinois \\ Urbana, Illinois 61801
}

\section{INTRODUCTION}

The rigorous analysis of microstrip geometries in a planar medium via the method of moments (MoM) requires the use of the Green's functions either in the spectral domain or in the spatial domain. The spectral domain Green's functions of HED, HMD, VED and VMD can be obtained in closed-forms in multilayer media, while the associated spatial domain Green's functions can be written in terms of the Sommerfeld integrals [1]. Since the application of the MoM transforms an operator equation into a matrix equation, the calculation of the matrix elements, which involve the Green's functions in the corresponding domain, becomes an important issue for the computational efficiency of the technique. The matrix elements of the MoM are triple integrals in the spatial domain, one of which is the Sommerfeld integration over an infinite domain, and double integrals in the spectral domain. The evaluation of the matrix elements in either domain is quite time-consuming because of the oscillatory nature and slow convergence behavior of the integrands [2]. In the spatial domain, the Sommerfeld integral, which is the representation of the spatial domain Green's functions, is the source of the oscillatory nature and the slow convergence of the integrands in the MoM matrix elements. To circumvent this problem, one needs to obtain the Green's functions in the spatial domain more efficiently that by evaluating the Sommerfeld integral. Recently, an approach to casting the spatial domain Green's functions into closed forms has been developed for HEDs [2], [3], and extended to the sources of VED, HMD and VMD in a multilayer medium [4]. With the use of this approach, it was demonstrated that the computational efficiency of the $\mathrm{MoM}$ for the solution of the mixed potential integral equations can be improved significantly for planar microstrip geometries [5]. However, further investigation has shown that the improvement in the computational efficiency may not be achieved for three-dimensional geometries in planar layered media, simply by casting the spatial domain Green's functions into closed forms. This paper discusses the difficulties involved in using the spatial domain, closed-form Green's functions in the Method of Moments formulation for three-dimensional geometries and proposes a technique to improve the computational efficiency of the MoM.

\section{FORMULATION}

A three-dimensional microstrip geometry shown in Fig. 1 is considered as a typical example to help demonstrate the use of the closed-form, spatial domain Green's functions and to help devise an approach to make the application of the MoM computationally efficient. The mixed-potential integral equation for the geometry, shown in Fig.1, can be transformed into the following matrix equation with the use

* Supported in part by NATO's Scientific Affairs Division in the framework of the Science for Stability Programme.

0-7803-2009-3/94/\$4.00 1994 IEEE. 1354 


$$
\begin{aligned}
& \text { of the well-known MoM procedure; } \\
& \qquad \begin{array}{c}
\left.\sum_{n=1}^{N} I_{n x}\left[\left\langle T_{n^{\prime} x}, G_{x x}^{A} * B_{n x}\right\rangle+\frac{1}{\omega^{2}}<T_{n^{\prime} x}, \frac{\partial}{\partial x}\left(G_{x}^{q} * \frac{\partial}{\partial x} B_{n x}\right)\right\rangle\right]+ \\
\left.\sum_{m=1}^{M} I_{m z}\left[\frac{1}{\omega^{2}}<T_{n^{\prime} x}, \frac{\partial}{\partial x}\left(G_{z}^{q} * \frac{\partial}{\partial z} B_{m z}\right)\right\rangle\right] \quad n^{\prime}=1, \ldots, N
\end{array} \\
& \sum_{n=1}^{N} I_{n x}\left[\left\langle T_{m^{\prime} z}, G_{z x}^{A} * B_{n x}\right\rangle+\frac{1}{\omega^{2}}<T_{n^{\prime} z} \cdot \frac{\partial}{\partial z}\left(G_{x}^{q} * \frac{\partial}{\partial x} B_{n x}\right)\right]+\quad m^{\prime}=1, \ldots, M
\end{aligned}
$$

where $T_{n^{\prime} x}, T_{m^{\prime} z}$ are the testing functions, $B_{n x}, B_{m z}$ are the basis functions and the spatial domain Green's functions $G$ for the vector and scalar potentials. It would be useful to list below the locations of the observation point $\mathrm{z}$ (domain of the testing functions) and the source point $z$ (domain of the basis functions) for each of the Green's functions appearing in (1).

$G^{A}{ }_{x x}\left(x-x^{\prime}, y-y^{\prime}, z-z^{\prime}\right)$ where the observation and source points are both located in the plane of the horizontal conductor, $\mathrm{z}=\mathrm{z}^{\prime}=\mathrm{d}_{\mathrm{i}}$-h in Fig. 1 .

$G^{A} x_{x}\left(x-x^{\prime}, y-y^{\prime}, z-z^{\prime}\right)$ where the source point $z^{\prime}$ is located in the plane of the horizontal conductor $\left(z^{\prime}=d_{i}-h\right)$ while the observation point $z$ varies from the ground plane to the plane of the horizontal conductor $\left(-\mathrm{d}_{-1}-\mathrm{h} \leq \mathrm{z} \leq \mathrm{d}_{\mathrm{i}}-\mathrm{h}\right)$.

$G q_{x}\left(x-x^{\prime}, y-y^{\prime}, z-z^{\prime}\right)$ where $z^{\prime}=d_{i}-h$, and $-d_{j-1}-h \leq z \leq d_{j}-h$. The second inner product term of Eq. 1 requires $z=z^{\prime}=d_{i}-h$, but in the second inner-product term of Eq. 2, one needs to evaluate the Green's function in the range $-d_{j-1}-h \leq z \leq d_{i}-h$.

$\mathrm{G}^{A} \mathrm{zx}\left(\mathrm{x}-\mathrm{x}^{\prime}, \mathrm{y}-\mathrm{y}^{\prime}, \mathrm{z}-\mathrm{z}^{\prime}\right)$ where $-\mathrm{d}_{\mathrm{i}-1}-\mathrm{h} \leq \mathrm{z}^{\prime} \leq \mathrm{d}_{\mathrm{i}}-\mathrm{h}$ and $-\mathrm{d}_{\mathrm{i}-1}-\mathrm{h} \leq \mathrm{z} \leq \mathrm{d}_{\mathrm{i}}-\mathrm{h}$.

$\mathrm{Gq}_{\mathrm{x}}\left(\mathrm{x}-\mathrm{x}^{\prime}, \mathrm{y}-\mathrm{y}^{\prime}, \mathrm{z}-\mathrm{z}^{\prime}\right)$ where $-\mathrm{d}_{\mathrm{i}-1}-\mathrm{h} \leq \mathrm{z}^{\prime} \leq \mathrm{d}_{\mathrm{i}}-\mathrm{h}$ and $-\mathrm{d}_{\mathrm{i}-1}-\mathrm{h} \leq \mathrm{z} \leq \mathrm{d}_{\mathrm{i}}-\mathrm{h}$.

The difficulties involved in the use of the closed-form Green's functions in conjunction with the MoM originate from the way the spectral domain Green's functions are cast into the closed forms. Therefore, the spectral domain Green's functions used in this formulation are given in the source layer, which is assumed to be layer- $\mathrm{i}$, as

$$
\begin{aligned}
& \tilde{G}_{x x}^{A}=\frac{\mu_{i}}{2 j k_{z i}}\left\{e^{-j k_{z i}\left|z-z^{\prime}\right|}+A_{i} e^{j k_{z i}\left(z-z^{\prime}\right)}+C_{i} e^{-j k_{i}\left(z-z^{\prime}\right)}\right\} \\
& \tilde{G}_{z x}^{A}=\frac{-\mu_{i}}{2 j k_{z i}}\left\{\frac{k_{x} k_{z i}}{k_{\rho}^{2}}\left(A_{i}+B_{i}\right) e^{j k_{z i}^{\left(z-z^{\prime}\right)}}+\frac{k_{x} k_{z i}}{k_{\rho}^{2}}\left(D_{i}-C_{i}\right) e^{-j k_{z i}\left(z-z^{\prime}\right)}\right\}
\end{aligned}
$$

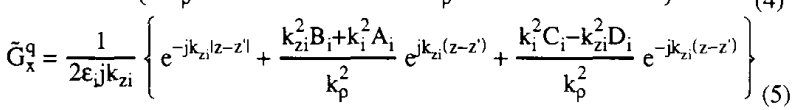

$$
\begin{aligned}
& \tilde{G}_{z z}^{A}=\frac{\mu_{i}}{2 j k_{z i}}\left\{e^{-j k_{z i}\left|z-z^{\prime}\right|}+A^{-} e^{j k_{z i}^{\left(z-z^{\prime}\right)}}+A^{+} e^{-j k_{z i}\left(z-z^{\prime}\right)}\right\} \\
& \tilde{G}_{z}^{q}=\frac{-1}{2 \varepsilon_{i} j k_{z i}}\left\{ \pm e^{-j k_{z i}\left|z-z^{\prime}\right|}+A^{-} e^{j k_{z i}\left(z-z^{\prime}\right)}-A^{+} e^{-j k_{z i}\left(z-z^{\prime}\right)}\right\} \quad z \lesseqgtr 0
\end{aligned}
$$


where the coefficients $A_{i}, B_{i}, C_{i}, D_{i}, A^{+}$and $A^{-}$are the functions of the generalized reflection coefficients, the parameters of the geometry and the source point $z^{\prime}$, which have been given in [4]. Note that the source points are in the layer i-1 for the lower part of the vertical conductor, so the subscripts $i$ in Eqs. (6) and (7) should be replaced by $\mathrm{i}-1$ when calculating the contribution of this part of the vertical conductor. In addition, if the observation points lie in a region different from the source points, then the amplitudes of the waves in the observation region are obtained recursively from the amplitudes of the waves in the source region [1].

In the process of casting the spectral domain Green's functions, given in Eqs. (3)(7), into closed forms, one needs to approximate the terms in the curly brackets by complex exponentials and then transform the spatial domain Green's functions in closed forms by using the Sommerfeld identity. The approximation by the complex exponentials can be performed by using the generalized pencil of functions (GPOF) method [6], which uses the sampled values of the terms in the curly brackets along a deformed path on the complex $k_{p}$-plane.

\section{DISCUSSION}

The elements of the MoM matrix for a typical three-dimensional geometry, and the corresponding Green's functions, have been given in the previous section, but the difficulties in using the closed-form Green's functions in Eqs. (1) and (2) have not been addressed as yet. As mentioned above, the difficulties arise from the setting of the observation and source points, $z$ and $z^{\prime}$, respectively, to constant values in these expressions. Since some of the Green's functions are required to be evaluated in the range of $z$ and/or $z$ ' values, alluded to above, the approximation procedure, GPOF, must be performed anew for each value of $z$ and $z$, rendering the entire procedure very time-consuming. This point could be better understood by examining, more closely, the inner product terms

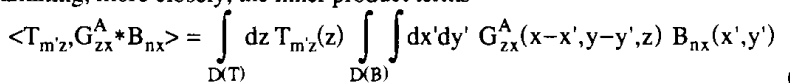

where $D(T)$ and $D(B)$ stand for the domain of testing and basis functions, respectively, and the vertical connection is assumed to be a thin wire for which $x$ and $y$ coordinates are fixed. We now face the following two difficulties: (i) the spectral domain representation of $\mathrm{G}^{\mathrm{A}} \mathrm{zx}$ has the term $\mathrm{k}_{\mathrm{x}}$, which cannot be written in terms of the sampled values of $k_{2}$ and $k_{p}$ alone; (ii) the GPOF method must be applied for each value of $\mathrm{z}$ in the outer integral. The first problem can be handled by excluding the $-\mathrm{jk}_{\mathrm{x}}$ term in the approximation and including its contribution in the spatial domain by differentiating with respect to $\mathrm{x}$, as follows

$$
\frac{\tilde{G}_{\mathrm{zx}}^{A}}{-j k_{\mathrm{x}}} \Leftrightarrow \mathrm{Z}_{\mathrm{zx}}^{\mathrm{A}} \text { then }\left\langle\mathrm{T}_{\mathrm{m}^{\prime}{ }^{\prime}}, G_{\mathrm{zx}}^{\mathrm{A}} * \mathrm{~B}_{\mathrm{nx}}\right\rangle=\left\langle\mathrm{T}_{\mathrm{m}^{\prime} \mathrm{z}}, \frac{\partial}{\partial \mathrm{x}} \mathrm{Z}_{\mathrm{zx}}^{\mathrm{A}} * \mathrm{~B}_{\mathrm{nx}}\right\rangle
$$

where the spatial derivative can be transferred over the basis functions. The second difficulty can be overcome by interchanging the order of integration, provided the basis and testing functions are so chosen that the involved integrals are uniformly convergent [7], and carrying out the integration over $z$ analytically for the spectra domain representation of the Green's function multiplied with the testing function. Next, the approximation method, GPOF, is applied to the resulting spectral domain function. This approach can be better understood by referring to the equation below; 
$<\mathrm{T}_{\mathrm{m}^{\prime} \mathrm{z}}, \mathrm{G}_{\mathrm{zx}}^{\mathrm{A}} * \mathrm{~B}_{\mathrm{nx}}>=\iint_{\mathrm{D}(\mathrm{B})} \mathrm{dx}^{\prime} \mathrm{d} \mathrm{y}^{\prime} \frac{\partial}{\partial \mathrm{x}} \boldsymbol{F}^{-1}\left\{\int_{\mathrm{D}(\mathrm{T})} \mathrm{dz} \mathrm{T}_{\mathrm{mz}}(\mathrm{z}) \frac{\tilde{G}_{\mathrm{zx}}^{\mathrm{A}}\left(\mathrm{k}_{\mathrm{p}}, \mathrm{z}\right)}{-\mathrm{jk} \mathrm{k}_{\mathrm{x}}}\right\} \mathrm{B}_{\mathrm{nx}}\left(\mathrm{x}^{\prime}, \mathrm{y}^{\prime}\right)$

where the integral in the curly bracket is evaluated analytically, see Eq. (5), and the GPOF is applied to the resulting equation to find the spatial domain representation. For the inner-product terms involving both the $z$ and $z^{\prime}$ integrations, these integrals can be performed analytically by using the procedure described above, and subsequently applying the GPOF algorithm. Note that the spectral representations of the Green's functions are exponential functions of $z$ and $z$, and this permits us to carry out the $\mathrm{z}$ and $\mathrm{z}$ integrations analytically.

\section{References:}

[1] W. C. Chew, Waves and Fields in Inhomogeneous Media. New York: Van Nostrand Reinhold, 1990.

[2] M. I. Aksun and R. Mittra, "Derivation of closed-form Green's functions for a general microstrip geometries," IEEE Trans. Microwave Theory Tech. a general microstrip geometries," IEE
vol. 40, pp. 2055-2062, Nov. 1992.

[3] Y. L. Chow, J. J. yang, D. H. Fang, and G. E. Howard," Closed-form spatial Green's function for the thick substrate," IEEE Trans. Microwave Theory Tech., vol. 39, pp. 588-592, Mar. 1991.

[4] G. Dural and M. I. Aksun," Closed-form Green's functions for general sources and stratified media," submitted for publication.

[5] M. I. Aksun and R. Mittra," Spurious radiation from microstrip interconnects," IEEE Trans. Electromagn. Compat., vol. 35, pp. 148-158, May 1993.

[6] Y.Hua and T. Sarkar," Generalized pencil-of-function method for extracting poles of an EM system from its transient response," IEEE Trans. Antennas Theory Propagat., vol. 37, pp. 229-234, Feb. 1989.

[7] M. I. Aksun and R. Mittra," Choices of expansion and testing functions for the method of moments applied to a class of electromagnetic problems," IEEE Trans. Microwave Theory Tech., vol. 41, pp. 503-509, Mar. 1993.

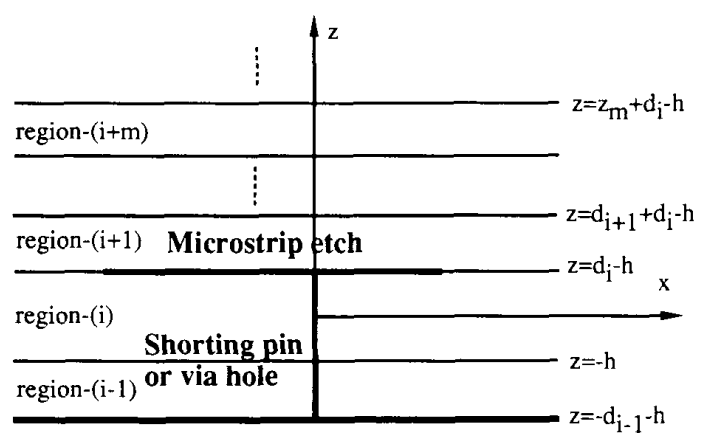

PEC ground plane

Fig.1. A typical three-dimensional microstrip geometry 